Service social

\title{
Les groupes de femmes : outils de changement personnel et de développement
}

\author{
Alice M. Home
}

Volume 37, numéro 1-2, 1988

Par-delà les barrières des sexes

URI : https://id.erudit.org/iderudit/706386ar

DOI : https://doi.org/10.7202/706386ar

Aller au sommaire du numéro

\section{Éditeur(s)}

École de service social de l'Université Laval

ISSN

1708-1734 (numérique)

Découvrir la revue

Citer cet article

Home, A. M. (1988). Les groupes de femmes : outils de changement personnel et de développement. Service social, 37(1-2), 61-85.

https://doi.org/10.7202/706386ar
Résumé de l'article

Cet article traite des groupes de femmes axés sur le partage du vécu, la résolution de problèmes ou la croissance personnelle. On y retrouve les groupes de thérapie/croissance animés par des intervenantes féministes, les groupes axés sur le soutien et l'aide mutuelle entre les membres et ceux qui ajoutent, à l'une ou l'autre de ces orientations, un objectif de conscientisation. Parmi les obstacles que ces groupes peuvent rencontrer, on retrouve la difficulté de concilier le soutien et le défi ainsi que l'autonomie et la dépendance, de même que certains problèmes reliés au choix d'un leadership approprié, au rythme de changement à adopter ainsi qu'aux différences culturelles entre les membres. Pour surmonter ces obstacles, il faut faire face aux contradictions entre l'idéologie féministe et la réalité quotidienne des femmes, et accorder une attention particulière à la planification des groupes. 
HOME, Alice M., professeure à l'École de service social de l'Université de Montréal.

\section{Les groupes de femmes: outils de changement personnel et de développement}

\section{Alice M. Home}

La thérapie traditionnelle n'aide pas les femmes à sortir des rôles stéréotypés ; au contraire, elle a contribué à la reproduction des rapports sexistes. La contestation de la thérapie traditionnelle a mené à l'élaboration du modèle d'intervention féministe qui vise à libérer les femmes des rôles qui les empêchent de réaliser leur potentiel (Corbeil et al., 1983). Cette intervention se fait souvent en groupe afin de promouvoir la solidarité et l'autonomie tout en réduisant l'isolement et la dépendance. Avant même que ce modèle d'intervention n'apparaisse, les féministes avaient mis sur pied des groupes autonomes leur permettant de réaliser des objectifs diversifiés, allant de la thérapie et de l'entraide jusqu'à l'action sociale.

Tout groupe féministe recherche, dans une certaine mesure, le changement personnel et social. Cet article se concentrera cependant sur les groupes qui visent le changement personnel et le développement chez les membres, étant donné que les groupes axés surtout sur la conscientisation et le changement social ont été discutés ailleurs (Home, 1983, 1980). Pour identifier les caractéristiques des groupes qui font l'objet de cet article, nous utiliserons des écrits descriptifs d'expériences, des énoncés théoriques, des recherches et des interventions faites dans divers milieux.

Nous commencerons en expliquant pourquoi les femmes choisissent de se regrouper entre elles. Nous montrerons en quoi les groupes axés sur le changement personnel et le développement se distinguent de tout autre groupe de femmes. Nous présenterons ensuite deux soustypes: les groupes de thérapie/croissance et de soutien/entraide, propos que nous complèterons par une brève discussion de ceux qui combinent l'un ou l'autre de ces objectifs et un but de conscientisation. 
Nous terminerons en soulevant certains obstacles que peuvent rencontrer ces groupes ainsi que quelques principes d'animation et d'intervention susceptibles de favoriser leur réussite.

\section{Pourquoi se regrouper entre femmes?}

Les femmes se sont d'abord regroupées pour des raisons idéologiques. Les groupes de conscientisation (CR), ${ }^{1}$ premiers groupes à paraître dans les années 1960, ont permis aux femmes de découvrir une solidarité, à partir des liens entre leur vécu personnel et l'oppression commune, et parfois d'agir individuellement ou collectivement contre cette oppression. Ces groupes étaient structurés de façon à encourager les femmes à dépasser les comportements de dépendance ou de passivité et à acquérir de nouvelles compétences de leadership. Même si le changement personnel était perçu comme un moyen d'amener les membres à s'impliquer dans le mouvement féministe, plusieurs femmes ont réalisé des changements importants dans l'estime de soi et la confiance (Home, 1980).

Toute population opprimée doit, dans un premier temps, découvrir l'oppression et la combattre (Freire, 1970). Mais en plus de ces raisons idéologiques, il existe également des raisons pratiques justifiant un regroupement. Chez certaines populations de femmes, la nature de leur situation commune exige qu'elles se regroupent sans hommes. Les groupes de femmes chefs de famille doivent permettre à leurs membres d'exprimer sans gêne leur colère contre les hommes, alors que les femmes violentées doivent se retrouver entre elles non seulement pour réduire l'isolement, mais aussi pour des raisons de sécurité (Gottlieb et al., 1983).

De plus, certaines recherches laissent entendre que les groupes mixtes peuvent reproduire les rapports sexuels traditionnels. Les groupes de croissance sont plus bénéfiques aux hommes car, contrairement aux femmes, ceux-ci y partagent davantage leurs sentiments que lorsqu'ils sont regroupés entre eux (Aries, 1976; Wheelan, 1980). Les femmes ont tendance à suivre ou à appuyer les interventions des hommes (Wodak, 1981). De plus, les groupes mixtes ont tendance à choisir un leader masculin, même si les femmes démontrent plus de capacité de leadership parce qu'elles réduisent la distance sociale entre elles et les membres, et parce qu'elles sont moins préoccupées que les hommes par le pouvoir (Megargee, 1969 dans : Denmark, 1977 ; Wodak, 1981).

En l'absence des hommes, les femmes adoptent des rôles moins stéréotypés et des attitudes plus féministes (Toder, 1980). Elles s'expriment 
de façon plus affirmative et moins défensive que lorsqu'elles font partie de groupes mixtes (Aries, 1976). Les groupes de femmes peuvent augmenter les comportements androgynes (équilibrant les caractéristiques dites "masculines" et "féminines") chez leurs membres (Mausert, 1979), et ainsi les outiller pour répondre de façon appropriée plutôt que stéréotypée aux changements de situation tels que le veuvage, la séparation ou un retour sur le marché du travail (Maracek, 1979).

Il existe toutefois certains groupes, notamment ceux axés sur l'affirmation de soi, qui aident les femmes, qu'il y ait ou non des hommes (Eichenbaum, 1978 ; Wilmuth, 1979). D'une part, les femmes ont besoin d'acquérir cette compétence et celle de la résolution de problèmes, et il n'est donc pas surprenant qu'elles profitent de ce type de groupe mixte plus que de ceux axés sur les relations interpersonnelles où elles détiennent déjà des habiletés (Wheelan, 1980); d'autre part, il est probable que les hommes impliqués dans les groupes d'affirmation de soi n'assument pas un rôle dominateur qui écrase les membres féminins.

II ressort de ces recherches, peu nombreuses, que dans bien des situations, les femmes ont raison de vouloir se regrouper entre elles. Dans la section suivante, nous décrivons les traits communs des groupes de femmes pour ensuite présenter les caractéristiques des groupes axés sur le changement personnel et le développement.

\section{Traits communs des groupes de femmes et caractéristiques des groupes axés sur le changement personnel et le développement}

Il existe maintenant tout un éventail de groupes de femmes. Les groupes de conscientisation (CR) existent toujours, quoiqu'ils se soient spécialisés de façon a favoriser la prise de conscience d'une double oppression en tant que femme noire, handicapée, lesbienne, âgée ou issue de la classe populaire. Certains groupes combinent la conscientisation et la poursuite d'un autre objectif comme l'apprentissage, alors que d'autres se concentrent sur le soutien, l'entraide, la thérapie ou sur la revendication de changements dans la condition des femmes.

\section{Traits communs à tout groupe de femmes}

Ces divers groupes partagent toutefois quelques caractéristiques communes. On retrouve partout une double préoccupation pour le 
changement personnel et social, reflet du principe féministe affirmant que "le privé est politique". Contrairement aux autres mouvements sociaux qui craignent que la recherche de solutions individuelles nuise à la solidarité, le mouvement féministe a redéfini le changement personnel comme étant compatible avec le changement social :

"La confiance en nous-mêmes nous donne la force de lutter pour le changement social et de faire face aux problèmes et à l'instabilité qui accompagnent souvent cette lutte. Seules celles qui prennent la responsabilité pour elles-mêmes peuvent créer les changements énormes nécessaires dans notre société. Seul un mouvement... qui appuie les efforts individuels pourrait réussir à changer la société." (Bunch, $1975: 6)$

Cependant, l'application de ce principe n'est pas uniforme dans tous les groupes. Ceux qui visent surtout à changer les lois, les attitudes ou les politiques touchant les femmes peuvent augmenter l'estime de soi et diminuer les sentiments d'impuissance chez leurs membres, mais ces effets sont secondaires (Levens, 1968 ; Rothman, 1976). De la même façon, l'objectif d'une action sociale reste plutôt un idéal qu'une pratique systématique dans bien des groupes qui recherchent surtout le changement personnel (Corbeil et al., 1983). Ces derniers se limitent souvent à discuter l'impact, sur les membres, de la socialisation et de la dévalorisation des femmes, de l'exploitation sexuelle ou de la tolérance sociétale de la violence envers celles-ci (Gottlieb et al., 1983). Certaines auteures déplorent que les groupes féministes ne passent pas tous à l'action (Gingras, 1986; Larsen et Cammaert, 1985). D'autres soulignent qu'il faut tenir compte, d'une part, de l'autonomie et des besoins des membres dans un groupe donné, et d'autre part, d'un équilibre entre les deux types de changements (Home, 1980, 1983).

Peu importe que la priorité soit mise sur l'action personnelle ou sociale, la majorité des groupes féministes essaient de réduire l'isolement et la compétition qui empêchent les femmes de comprendre leur vécu d'oppression, de se valoriser et de se respecter en tant que femmes (Gottlieb et al., 1983). En même temps que ces groupes soutiennent les membres dans leur comportement d'affirmation à l'extérieur du groupe, ils leur fournissent l'occasion, à l'intérieur du groupe, d'exercer leurs habiletés de leadership. On espère alors promouvoir la prise en charge par les femmes de leur vie individuelle et de leur condition commune (Gottlieb et al., 1983). On utilise à ces fins des structures égalitaires et informelles, et lorsqu'il y a des animatrices désignées, celles-ci démystifient leur rôle en même temps qu'elles partagent avec les membres les tâches et les habiletés d'animation (Cardin et Home, 1983; Home, 1980). 
Les groupes peuvent aider leurs membres à être plus réalistes quant à la responsabilité qu'ils doivent assumer dans leurs problèmes personnels. Une fois que ces femmes ont reconnu les racines politiques de leurs difficultés, elles sont plus en mesure de distinguer les aspects qu'elles peuvent changer individuellement de ceux requérant une action collective. Les femmes chefs de famille découvrent, par exemple, que la société leur impose de lourdes responsabilités sans leur offrir le soutien requis pour y faire face. Elles constatent alors qu'elles ne sont pas inadéquates même si elles se sentent parfois écrasées (Gottlieb et al., 1983).

\section{Traits spécifiques aux groupes axés sur le changement personnel et le développement}

Ces groupes se distinguent par la priorité accordée à la résolution de problèmes vécus par leurs membres ou à la poursuite de leur croissance personnelle. Comme les membres ne font pas toujours de liens entre leur situation individuelle et la condition générale des femmes, ces groupes encouragent une certaine analyse sociale des racines politiques à la source des problèmes identifiés (Wolman, 1976). Contrairement à la majorité des groupes de femmes, ceux-ci ont souvent une ou deux animatrices désignées qui peuvent éventuellement restreindre leur rôle ou se retirer du groupe.

Différents types de changements peuvent se produire chez les membres de ces groupes au plan des sentiments, des comportements ou des attitudes. Beaucoup de femmes modifient leurs sentiments à l'égard d'elles-mêmes et se sentent ainsi plus confiantes, moins coupables, plus solides dans leur identité et moins dépendantes des relations avec les hommes et les enfants. Cette transformation personnelle semble être un prérequis aux autres types de changements (Farquharson, 1975), surtout chez les femmes, à cause de la socialisation qui peut leur laisser des sentiments d'incompétence, d'impuissance et de dépendance.

D'autres changements affectent particulièrement les comportements. En plus de se sentir plus confiante, la femme peut démontrer des comportements plus affirmatifs ou plus autonomes. Ces changements peuvent se répercuter dans l'organisation et l'entente familiales: par exemple, une femme n'avait plus peur de demander une journée de liberté chaque fin de semaine, après avoir découvert son besoin et son droit d'avoir du temps pour elle. D'autres adoptent des comportements plus affirmatifs à l'extérieur du foyer (chez le médecin, au bureau, etc.) ou se réorientent vers les études ou le marché du travail. 
Un troisième type de changements se manifeste dans l'attitude par rapport au rôle des femmes, mais il peut se traduire également dans un comportement nouveau. Par exemple, une femme interviewée sur son vécu dans un groupe de conscientisation a reconnu l'irréalisme du mythe de la " superfemme " qui fait tout, parfaitement, en même temps. Elle a également réfléchi sur la signification personnelle du mot "féministe" avant de prendre une décision importante pour elle :

"J'ai décidé que je n'ai plus besoin d'être la femme et la mère parfaites tout en travaillant à l'extérieur. Ce n'est pas une solution réaliste pour moi. La société est trop exigeante envers les femmes. Je peux être féministe tout en choisissant de rester au foyer pendant que mes enfants sont très jeunes."

Tout groupe féministe axé sur le changement personnel ou le développement peut connaître ces trois formes de modifications. Cependant, la structure de certains groupes semble favoriser des transformations spécifiques chez leurs membres. Les groupes de thérapie/croissance semblent promouvoir à la fois des changements dans les comportements et les sentiments, alors que les groupes de soutien/entraide restent plus souvent au niveau de l'estime de soi et du contrôle personnel (Sprei-ott, 1980 ; Vandervoet, 1978). Lorsque la conscientisation figure parmi les objectifs, le changement d'attitude prend une plus grande importance. Dans la section suivante, nous discuterons de ces soustypes séparément, même s'ils se combinent souvent dans la pratique.

\section{Les groupes de thérapie/croissance}

Cette catégorie inclut des groupes diversifiés qui partagent l'objectif d'aider leurs membres à modifier leurs sentiments, leurs comportements, leurs relations interpersonnelles et parfois leurs attitudes (Walker, 1981). D'habitude, ces groupes sont sous la responsabilité d'une ou deux intervenantes qui, souvent, ont une formation professionnelle et s'inspirent des principes de l'intervention féministe. Nous décrirons, dans la section suivante, les objectifs, le programme, le leadership et les techniques caractéristiques de ces groupes, en distinguant, lorsque approprié, entre les groupes de thérapie et ceux axés sur la croissance. La même structure sera employée dans la section sur les groupes de soutien/entraide afin de faciliter la compréhension du texte. 


\section{Objectifs et programme}

Les groupes de thérapie ont comme objectifs de favoriser la résolution de problèmes personnels identifiés par les membres, promouvoir leur resocialisation ou les aider à acquérir des attitudes et des compétences qui puissent les soutenir dans leurs efforts de dépasser les rôles traditionnels (Sturdivant, 1980). L'orientation et le programme de chaque groupe dépendent à la fois de la composition de celui-ci et de son objectif spécifique.

Les groupes dont les membres sont très dépendants ou défavorisés mettent d'abord l'accent sur la resocialisation et la revalorisation, ainsi que sur l'acquisition de compétences aptes à réduire la passivité et la dépendance. Un groupe composé de femmes peu scolarisées et longtemps absentes du marché du travail mettait l'accent sur la connaissance et l'affirmation de soi. Compte tenu du peu de confiance et d'autonomie des membres, les objectifs d'acquisition de compétences concrètes en vue d'un retour à l'emploi ont joué un rôle secondaire, et la durée du groupe (seize rencontres) était plus longue que chez la clientèle régulière de l'organisme. L'intervenante professionnelle a utilisé divers exercices pour aider ces femmes à situer leur marginalité par rapport au marché de l'emploi dans un contexte de dévalorisation sociétale des femmes et de leur travail (Bernier, 1984).

On retrouve dans cette catégorie des groupes composés de femmes

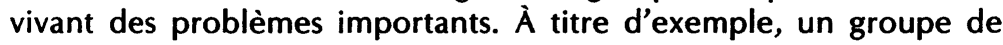
femmes déprimées, âgées de 45 à 65 ans, a été mis sur pied suite à un premier traitement en milieu psychiatrique. Les intervenantes ont incité les membres à travailler sur leurs difficultés personnelles en vue de prendre en charge leur vie, dans un contexte où elles les ont encouragées à prendre conscience des limites des rôles traditionnels (Demers, 1984).

Les groupes de femmes victimes de violence conjugale se font de plus en plus nombreux. Comme ces femmes ont été longtemps dévalorisées et isolées, on travaille d'abord à augmenter l'estime de soi et la capacité affirmative, dans un climat de solidarité et de soutien (Labrosse, 1987). D'ailleurs, le choix de ces objectifs spécifiques correspond aux écrits sur le sujet qui établissent une interdépendance entre l'intégration du stéréotype féminin, la tolérance de la violence et la difficulté à vivre la solitude, chez les femmes qui se définissent surtout à partir de la relation avec un homme. On travaille le vécu spécifique de la femme violentée à partir de ces thèmes : violence, sexualité, colère et notion de victime (Larouche et Vaillant, 1986).

D'autres groupes, s'inspirant davantage des groupes mixtes de croissance personnelle et composés de femmes sans problème spécifique, 
tentent de faire ressortir les traits de personnalité dont l'expression a été freinée par les contraintes du rôle traditionnel. Ces groupes permettent alors à leurs membres de devenir plus androgynes (Meador et al., 1972). Un de ces groupes, composé d'étudiantes en milieu universitaire, met l'accent sur la discussion de sujets " tabous", sur l'estime de soi et sur les modifications à apporter aux relations interpersonnelles tant avec les hommes qu'avec les femmes (Killeen et Jacobs, 1976).

Certaines auteures affirment que la femme qui prend conscience de son oppression lorsqu'elle est en contact avec d'autres femmes, va nécessairement changer, alors que d'autres considèrent qu'on ne peut pas évaluer le changement réalisé à moins que la femme ne spécifie, dans un contrat thérapeutique, les comportements observables qu'elle veut modifier (Wyckoff, 1971). Certains groupes, notamment ceux axés sur l'affirmation de soi, utilisent des techniques de la thérapie behaviorale pour apprendre aux femmes à acquérir de nouvelles façons d'agir. Ces derniers groupes enseignent à leurs membres comment protéger leurs droits tout en respectant ceux des autres et comment exprimer de façon directe, honnête et ouverte leurs sentiments, idées et croyances (Jakubowski-Spector, 1973). D'autres groupes visent l'acquisition de compétences en résolution de problèmes, tout en maintenant leur objectif de modification de comportement. Dans ces groupes, l'intervenante aide les membres à définir leur problème, à déterminer la façon dont ils désirent changer et à réaliser le changement étape par étape, le tout dans un climat de soutien. À chaque rencontre, les membres partagent leur vécu quant au processus de changement (Wyckoff, 1977). Les transformations vécues à l'extérieur du groupe, de même que les échanges sur les réussites et les échecs, renforcent les femmes dans leurs objectifs de changement (Home, 1980).

\section{Leadership}

La majorité des groupes de thérapie/croissance sont animés par des intervenantes, même si des équipes hommes-femmes peuvent parfois être utiles pour illustrer un modèle égalitaire de partage de tâches (Rounsaville et al., 1979). Des recherches indiquent que les thérapeutes féminines peuvent servir de modèle androgyne à cause de l'expression ouverte de leurs sentiments et de leur compétence, jumelées à une attitude de soutien (Walker, 1981).

Le rôle de leadership ressemble sous certains aspects à celui observé dans des groupes mixtes axés sur le changement personnel. Même si ce rôle varie selon l'objectif spécifique du groupe, les leaders sont portés à y être plus directifs que dans d'autres types de groupes. Ils perçoivent 
les membres comme étant peu autonomes, et moins du tiers d'entre eux partagent avec les participants les tâches de déterminer les objectifs ou de planifier le programme (Darveau-Fournier et Home, 1983). Dans un groupe de changement personnel, le leader assume d'habitude un rôle d'enseignant (qui se concentre sur un contenu planifié d'avance) ou de thérapeute qui prend la responsabilité de planifier et d'animer le groupe et d'aider directement les membres à résoudre leurs problèmes (Darveau-Fournier et Home, 1983).

On retrouve ces rôles assez souvent chez les leaders des groupes de femmes. Le rôle de thérapeute se manifeste plus particulièrement dans les groupes où les membres sont peu autonomes (Lang, 1972), lorsqu'ils ont des problèmes comme l'alcoolisme qui durent depuis longtemps ou quand ils sont en état de crise. Le rôle d'enseignant se retrouve plus particulièrement dans les groupes qui visent l'acquisition d'un nouveau comportement comme l'affirmation de soi. Cependant, comme les féministes craignent qu'une professionnelle risque, de par sa formation et son statut de leader, de nuire à la prise en charge par le groupe, les intervenantes essaient de jouer un rôle moins central que dans des groupes mixtes à objectifs semblables. Dans les groupes de femmes, on met plus d'accent sur l'aide mutuelle entre les membres que dans les groupes mixtes, et le leader peut combiner le rôle de thérapeute ou d'enseignant avec celui, plus périphérique, de facilitateur d'aide mutuelle (Cardin et Home, 1983). La facilitateure favorise l'échange et la mutualité d'aide ainsi que le partage d'autorité dans l'animation et la prise de décision. Ainsi, dans le groupe de femmes déprimées, les intervenantes décrivent ainsi le rôle de facilitateure et de thérapeute: tenter de répondre aux besoins individuels des membres, être un modèle de communication, offrir une rétroaction (thérapeute), suggérer des activités, participer aux discussions et apporter des ressources matérielles (facilitateure) (Haussmann et Halseth, 1983). Ce rôle combiné est plus conforme à l'idéologie féministe qu'un rôle central.

\section{Techniques}

Les techniques employées dans ces groupes sont éclectiques car on les a empruntées à divers modèles d'intervention tels que la gestalt, les approches corporelles et la thérapie behaviorale (Corbeil et al., 1983) De plus, elles sont adaptées aux objectifs spécifiques du groupe. En plus d'utiliser les techniques de tout groupe féministe (discussion, partage de vécu), ces groupes mettent l'accent sur la révélation personnelle, les sujets " tabous » et le développement de compétences interpersonnelles entre femmes (Meador et al., 1972). 
Certains groupes se limitent à quelques techniques alors que d'autres emploient des moyens très diversifiés. Les groupes d'affirmation de soi se concentrent sur les techniques behaviorales en liant l'acquisition de compétences à une analyse de la socialisation des femmes (Gottlieb et al., 1983). Parmi ces procédés, on retrouve l'établissement d'une liste, par ordre de difficultés, des situations qui demandent un comportement affirmatif. On combine cette technique avec la répétition behaviorale, où les femmes pratiquent des comportements affirmatifs suivis d'une rétroaction du groupe, en commençant par des situations moins difficiles, par exemple, refuser une invitation. Les devoirs à faire entre les réunions aident les femmes à intégrer les nouveaux comportements appris (Butler, 1976).

D'autres groupes se servent d'une diversité de techniques. Le programme varié d'un groupe de femmes battues inclut l'entraînement à l'affirmation de soi, l'utilisation du vidéo, le travail de l'imaginaire et des sorties de groupe (Larouche et Vaillant, 1986). Un groupe pour patients psychiatriques en centre de jour s'est servi entre autres de la musique, de la lecture d'articles et d'histoires, de la préparation par chaque femme de son histoire de vie, pour favoriser un partage avec les autres membres (Adolph, 1983). Un groupe composé de femmes déprimées en milieu rural a combiné les techniques cognitives, l'entraînement à l'affirmation et les techniques de conscientisation. Les intervenantes ont utilisé divers procédés : remue-méninges, brèves séances de cours, jeux de rôle et exercices avec des phrases incomplètes (ex. : " Je me suis sentie heureuse/déprimée cette semaine lorsque... ") (Haussmann et Halseth, 1983).

Les groupes combinent souvent exercices structurés et partage du vécu. Souvent, les exercices choisis font appel à la créativité. Une activité de réchauffement, par exemple, consiste à demander à chaque femme de dessiner une forme qui représente sa vie. Elle ajoute ensuite d'autres dessins exprimant les événements critiques qu'elles a vécus et elle explique au groupe la signification de son dessin (WCREC, 1985).

En plus de ces techniques diversifiées, les intervenantes utilisent certaines habiletés plus spécifiques à l'intervention féministe. À partir de la théorie et de la recherche, Russell (1984) a identifié et opérationnalisé cinq techniques permettant d'évaluer le degré d'actualisation dans une intervention féministe donnée. Étant donné que Russell est la seule auteure à avoir suffisamment explicité ces techniques pour que les intervenantes puissent les apprendre, nous les décrirons en indiquant une application en milieu québécois.

L'analyse sociale consiste à comprendre, avec les femmes, comment elles se sont accommodées des contraintes sociales ayant eu ou ayant une influence sur leur comportement. L'intervenante aide les femmes à 
identifier les barrières intérieures et extérieures qui contribuent à leur situation problématique actuelle, de même qu'à relever les aspects politiques de leurs problèmes personnels (Russell, 1984). Dans un groupe de femmes aînées, par exemple, l'intervenante a aidé les membres à faire le lien entre leur difficulté à parler devant un groupe et le rôle traditionnel qui les a confinées au foyer (Hébert, 1985).

Une deuxième technique qu'on retrouve particulièrement dans les groupes de croissance et de thérapie est la rétroaction comportementale, qui consiste à décrire les dires ou les comportements des individus sans les juger ou les étiqueter. L'intervenante vise à faire ressortir les contradictions entre ce que la femme dit et ce qu'elle fait, en rapport avec son rôle de femme (Russell, 1984).

L'encouragement des comportements androgynes permet à la femme de se concentrer sans culpabilité sur un auto-développement équilibré (Russell, 1984). Des groupes d'affirmation de soi et de résolution de problèmes se servent souvent de cette technique pour donner aux femmes la permission de développer de nouveaux comportements et des outils pour le faire. Dans un groupe de femmes qui pensaient retourner sur le marché du travail, l'intervenante s'est servi d'un vidéo pour leur présenter des métiers non traditionnels auxquels elles n'avaient pas pensé, soit à cause d'aptitudes négligées ou des rôles traditionnels (Bernier, 1984).

L'auto-révélation implique la capacité de dévoiler les caractéristiques personnelles pertinentes afin d'aider les femmes à progresser dans leur cheminement (Russell, 1984). Cette technique offre un modèle de rôle en plus de réduire la distance sociale entre l'intervenante et les autres femmes dans le groupe, favorisant ainsi une relation plus égalitaire entre elles. Toujours dans le groupe de femmes aînées, l'intervenante a exprimé sa propre gêne de parler devant un groupe et sa conviction qu'il en serait toujours ainsi (Hébert, 1985).

L'autre technique décrite par Russell (1984) est l'évaluation positive par laquelle l'intervenante aide la femme à reconnaître ses qualités dites "féminines " et à les évaluer de façon positive. Dans un groupe de femmes longtemps absentes du marché de l'emploi, les membres ont pris conscience de leurs qualités de douceur, de générosité, de délicatesse et d'écoute. De plus, elles ont évalué l'utilité possible de ces qualités pour occuper certains postes à l'extérieur du foyer (ex. : préposée aux personnes âgées). Ces femmes ont aussi dressé une liste de tâches exécutées à la maison (préparer le budget, faire la liste pour l'épicerie, etc.) pour en faire ressortir les qualités qui s'y rattachent, par exemple le sens de l'organisation (Bernier, 1984).

En terminant cette section, il faut souligner que quelques-unes de ces techniques peuvent se retrouver également dans certains groupes 
mixtes (l'auto-révélation par exemple) et que le choix des techniques à utiliser doit tenir compte de la composition et de l'évolution de chaque groupe. Nous reviendrons sur ce point dans la dernière partie de l'article. Nous nous tournons maintenant vers les groupes de soutien/ entraide.

\section{Les groupes de soutien/entraide}

Plusieurs caractéristiques des groupes de thérapie / croissance sont partagées par les groupes de soutien/entraide. Dans cette section, nous ne traiterons que des aspects spécifiques à ces derniers afin d'éviter la répétition. Nous discuterons simultanément des groupes de soutien et d'entraide à cause de leurs similarités, tout en soulignant, au besoin, leırs différences. Les groupes de soutien mettent en présence des personnes qui veulent se donner du support pour faire face à une situation stressante commune (Rosenberg, 1984). Les groupes d'entraide sont de petits groupes autonomes qui travaillent sur un problème commun à leurs membres en utilisant l'aide mutuelle et l'action collective (Farquharson, 1975).

\section{Objectifs}

Les objectifs de ces groupes se distinguent de ceux des groupes de thérapie/croissance en privilégiant le développement plutôt que le changement personnel. On retrouve souvent parmi ces groupes des femmes qui veulent rencontrer d'autres femmes vivant une situation stressante similiare à la leur, afin d'échanger et de se donner du soutien mutuel. Dans ce type de groupes, les femmes ont moins souvent un problème spécifique à régler que dans les groupes de thérapie/ croissance. Alors que ces derniers mettent l'accent sur la résolution de problèmes et la réalisation de changement de comportement chez la personne, les groupes de soutien/entraide utilisent davantage le processus du groupe pour promouvoir l'assistance entre les membres et l'augmentation de l'estime de soi (Rosenberg, 1984).

Diverses situations peuvent provoquer la formation d'un groupe de soutien/entraide. Certains groupes visent à aider les femmes à traverser les crises temporaires et prévisibles de leur développement, par exemple, l'adolescence ou la maternité. Comme les réseaux familiaux et communautaires offrent peu de soutien dans la société actuelle, ces cellules peuvent fournir une nouvelle source d'aide qui réduit le stress 
associé à de telles transitions. D'autres groupes réunissent des femmes vivant une situation stressante commune comme la monoparentalité ou le veuvage. Être en groupe de soutien / entraide composé uniquement de femmes est très important pour celles qui sont dans des situations de transition qui les poussent à sortir du rôle féminin traditionnel, car les réseaux familiaux n'aident pas toujours de tels changements. Les femmes qui font un retour aux études ou à l'emploi et celles qui assument des responsabilités nouvelles, par exemple comme mères chefs de famille, ont besoin d'être soutenues dans leur nouveau rôle par d'autres femmes. À titre d'exemple, un groupe, composé de femmes au foyer ayant une formation universitaire, a offert du soutien aux membres, en plus de les informer sur les possibilités d'emploi ou de poursuite d'études en vue de recommencer leur carrière (Redfern et al., 1976).

Certains groupes peuvent se rencontrer pendant une courte période pour aider les femmes à traverser une transition ou une crise, alors que d'autres peuvent devenir un système de soutien à long terme. Un groupe à durée limitée réunissait toutes les femmes qui avaient déménagé dans une communauté donnée au cours de la dernière année. Comme la société s'est toujours attendu à ce que les femmes suivent les maris qui se déplacent pour leur carrière, on s'est peu occupé de l'impact des déracinements sur leur vie. Même si le groupe était composé de femmes de différents âges et d'origines ethniques variées et que cellesci pouvaient avoir vécu un ou plusieurs déménagements, toutes se sentaient isolées et cherchaient du soutien. Le groupe était animé par deux travailleuses sociales qui partageaient avec les membres l'expérience d'avoir été déracinées (Levin et al., 1980).

Dans une banlieue défavorisée, on a mis sur pied des groupes afin de donner aux femmes au foyer une occasion de sortir de la maison et de développer des intérêts à l'extérieur. La ville qu'elles habitaient avait un mauvais système de transport en commun, ce qui augmentait leur isolement, les confinant davantage dans leur rôle traditionnel. Des bénévoles ont animé ces groupes, en mettant l'accent sur la formation de liens entre femmes et sur le développement d'intérêts non reliés aux rôles de mère et d'épouse. Afin d'atteindre un grand nombre de femmes, le groupe restait ouvert à de nouveaux membres, même si les groupes se continuaient pendant plusieurs années.

Un groupe, formé de jeunes mères seules vivant de prestations de bien-être social, visait à leur offrir une occasion de partager leur vécu et de faire ensemble des activités récréatives. Pour ces jeunes femmes aux prises avec des contraintes sociales et financières importantes, ce groupe représentait un premier pas vers l'extérieur et a conduit plusieurs d'entre elles à un retour aux études. Compte tenu de l'isolement et du manque de ressources de ces femmes, l'intervenante (elle-même mère 
d'un jeune bébé) a offert beaucoup de soutien tant concret (gardiennage, transport) que socio-affectif.

Comme les groupes de thérapie, certains de ces groupes se forment pour aider les femmes aux prises avec des problèmes importants (toxicomanie, maladie mentale, violence conjugale) (WCREC, 1985). Toutefois, ces groupes de soutien/entraide sont moins structurés et appartiennent davantage à leurs membres que ceux axés sur la thérapie / croissance. À titre d'exemple, un groupe de femmes ayant vécu la violence conjugale, est toujours ouvert aux nouveaux membres qui veulent travailler sur leur situation. Il accueille des femmmes qui se situent à différents moments de leur vécu de violence, incluant celles qui ont quitté le conjoint violent. Compte tenu de la lourdeur de la problématique, une professionnelle aide l'animatrice qui a, elle aussi, vécu une relation abusive (Hartmann, 1983). Cependant, la professionnelle joue un rôle périphérique de personne-ressource qui appuie les leaders naturels en leur apportant une aide technique ou en leur offrant consultation et formation (Darveau-Fournier et Home, 1983). L'animatrice professionnelle aide ainsi au niveau du processus du groupe, des références au groupe, des liens avec d'autres intervenantes et au niveau des connaissances reliées à la problématique (Hartmann, 1983).

\section{Leadership}

La différence fondamentale entre ces groupes et ceux axés sur la thérapie/croissance se situe au niveau du leadership. Même si ces derniers visent un partage de vécu et d'autorité entre l'animatrice et les membres, celle-ci garde un certain contrôle de par son expérience et sa formation. Les groupes de soutien/entraide utilisent surtout l'aide mutuelle plutôt que professionnelle, et lorsque la responsabilité du groupe relève d'une intervenante ou d'une bénévole, celle-ci adopte un rôle périphérique de personne-ressource ou de facilitateure. Alors que les groupes de soutien ont souvent une animatrice qui réduit graduellement son rôle, les groupes d'entraide s'en remettent encore plus aux membres pour le contrôle du groupe. Comme le dit un collectif de femmes:

"Nous soulignons que les groupes d'entraide réussissent bien sans leader professionnel. Les femmes qui ont un vécu partagé avec un problème donné sont bien qualifiées pour se donner du soutien et de l'aide dans un groupe." (WCREC, $1985: 6$ )

L'expertise des leaders des groupes de soutien vient surtout de leur formation et de leur expérience professionnelle, alors que celle des 
membres d'un groupe d'entraide qui partagent le leadership relève de leur expérience et de leurs habiletés à faire face au problème (Rosenberg, 1984). Même si les professionnelles aident parfois les membres à mettre un groupe sur pied, ce sont les participants qui gardent le contrôle. II faut noter aussi que le groupe de soutien avec participation d'une intervenante peut se transformer ensuite en groupe d'entraide autonome (Home, 1985).

Une étude a démontré qu'il existe beaucoup de diversités chez ces groupes, non seulement dans l'utilisation qu'ils font d'intervenantes professionnelles, mais aussi par rapport au contenu et aux techniques employées (Women and Mental Health Project, 1976). Les groupes d'auto-santé, par exemple, sont axés sur la réappropriation de soi et de son corps en vue de réduire la dépendance au pouvoir médical (Corbeil et al., 1983). Ils font appel à des femmes non professionnelles pour enseigner l'auto-santé et animer les discussions (Kush-Goldberg, 1979). Parfois les groupes forment eux-mêmes leurs membres au leadership. Un centre de femmes a offert à sa clientèle une double formation aux rôles d'aidante et d'aidée. Après avoir acquis des habiletés comme l'écoute active et l'empathie, des petites équipes de trois à quatre femmes se sont rencontrées plusieurs fois pour les pratiquer (Glaser, 1976) en vue de devenir des personnes-ressources pour d'autres femmes.

\section{Programme et techniques}

Puisque le programme et les techniques utilisés par ces groupes sont reliés et qu'il existe peu de documentation sur le sujet, nous les avons réunis dans une même rubrique. Ces groupes utilisent plusieurs des techniques déjà discutées au sujet des groupes de thérapie/ croissance, y compris celles identifiées par Russell (1984). Cependant, les groupes de soutien et d'entraide se fient davantage sur des moyens informels, comme la discussion autour de thèmes, que sur des exercices structurés comme l'entraînement à l'affirmation de soi. Ces groupes combinent d'habitude le partage du vécu, la transmission d'informations (entre membres et entre personnes-ressources et groupe) et une réflexion (allant parfois jusqu'à l'action sociale) sur les causes politiques du vécu.

On y met plus l'accent sur le soutien et sur l'aide mutuelle entre les membres que dans les groupes de thérapie/croissance où l'aidante professionnelle utilisera parfois la confrontation dans un contexte de soutien. Le mécanisme de base, particulièrement dans les groupes d'entraide, est le "Helper therapy principle" selon lequel celle qui partage son expérience ou ses conseils avec d'autres femmes en profite 
aussi (Reissman, 1965). Comme les membres d'un groupe d'entraide se trouvent à différentes étapes dans leurs efforts pour faire face au problème qui leur est commun, les membres plus expérimentés peuvent offrir non seulement l'espoir de s'en sortir, mais aussi des conseils pratiques, spécifiques et orientés vers l'action (Borkman, 1976). Le groupe offre différentes façons de participer, de sorte qu'un nouveau membre d'un groupe de femmes battues, par exemple, peut surtout observer au début s'il a trop peur de parler (Hartmann, 1983).

Le groupe offre non seulement un soutien dans une situation difficile, mais aussi un renforcement lorsqu'une femme amorce des changements dans un sens plus "féministe". Dans ce sens, les groupes empruntent à la thérapie behaviorale la technique de renforcement sélectif dans un contexte où les changements sont favorisés à la fois par le soutien et l'influence du groupe. Levy (1979) a identifié les techniques et activités le plus souvent utilisées dans les groupes d'entraide : empathie, valorisation mutuelle, explication, partage, soutien moral, auto-révélation, renforcement positif, établissement d'objectifs personnels et ventilation. Les groupes observés par Levy étaient majoritairement mais non exclusivement féminins; toutefois, seuls certains d'entre eux avaient une orientation féministe explicite. Il faudra répéter cette recherche auprès des groupes de femmes pour vérifier si on retrouve toujours ces mêmes techniques. On peut remarquer la présence de quelques techniques qui ressemblent à celles décrites par Russell (1984) : auto-révélation, valorisation mutuelle (évaluation positive) et renforcement positif (semblable à l'encouragement des comportements androgynes). Toutefois, l'absence de l'analyse sociale parmi les techniques observées par Levy (1979) reflète peut-être une moins grande préoccupation, chez certains groupes d'entraide mixtes, pour la prise de conscience des racines politiques des problèmes vécus par les membres.

\section{Les groupes combinés}

Nous terminons cette section en parlant brièvement des groupes qui visent une juste mesure entre une prise de conscience de l'oppression des femmes et l'aide au développement personnel ou à la résolution de problèmes. Alors que, dans la plupart des groupes discutés dans cet article, la modification des attitudes est secondaire, la prise de conscience est l'objectif principal des groupes de conscientisation (CR). Certains groupes recherchent à la fois la conscientisation et le changement personnel en combinant les objectifs, le programme, les techniques et les modèles de leadership des deux types de groupe. 
Les groupes de conscientisation (CR) rejettent d'habitude l'idée d'un leadership professionnel, quoiqu'on y retrouve parfois des animatrices qui commencent par jouer un rôle de facilitateure/personneresssource pour ensuite assumer un rôle égal à celui des autres membres (Home, 1985). Dans les groupes à objectifs combinés, on retrouve plus souvent une intervenante qui assume un certain rôle d'animation ou de planification.

Ainsi, dans un groupe de femmes déprimées, on retrouve une intervenante professionnelle qui se sert d'exercices structurés en plus des techniques de conscientisation (échange et analyse du vécu par rapport à un thème) (Adolph, 1983). Un groupe de femmes en milieu populaire vise non seulement une augmentation de l'estime de soi, de l'autonomie et une identité plus solide en tant que personne mais aussi une prise de conscience de la double oppression des femmes de classe populaire (Gingras, 1983). Dans ce groupe, le changement personnel chez les membres s'est poursuivi par une action concrète dans le milieu, soit la création d'un centre de femmes. Des techniques variées y étaient employées, allant des approches corporelles et partage du vécu aux techniques de conscientisation de Paulo Freire (Gingras, 1983).

Un dernier exemple est un groupe d'aînées qui visaient non seulement à partager leur vécu de femme âgée et à prendre en charge leur vie mais aussi une prise de conscience de l'oppression spécifique de la femme âgée. Encore ici, on retrouve une intervenante professionnelle qui utilise à la fois les techniques de l'intervention féministe et celles de la conscientisation; cependant, ce groupe n'a pas entrepris d'action collective (Hébert, 1985).

\section{Favoriser la réalisation de changements : obstacles et stratégies}

Les groupes de femmes sont une alternative intéressante aux groupes mixtes ou à la thérapie traditionnelle. Toutefois, le fait de regrouper des femmes qui partagent les mêmes besoins ne garantit pas une aide mutuelle effective (Shulman, 1979). Si les groupes de femmes peuvent éviter certains problèmes caractéristiques aux groupes mixtes, ils risquent d'en rencontrer d'autres qui peuvent les empêcher de réaliser les changements recherchés. Nous discuterons, dans cette dernière section, de certains obstacles rencontrés par les groupes axés sur le changement personnel et le développement, ainsi que des stratégies pour les contourner. 
Plusieurs obstacles relèvent de contradictions entre une idéologie féministe parfois idéaliste et la réalité quotidienne des femmes qui font partie de ces groupes. On retrouve en particulier l'enjeu de l'autonomie versus la dépendance, avec les questions qui s'y rattachent et qui ont trait au rôle de leader, au rythme du changement et à l'importance à accorder au soutien ou au défi.

Le mouvement féministe met beaucoup l'accent sur l'acquisition de comportements plus autonomes souvent limités, dans le cas des femmes, par une socialisation à la dépendance et à la passivité. II faut toutefois adopter une attitude réaliste face à la difficulté que représente un tel changement. Si certaines femmes scolarisées de classe moyenne adoptent assez facilement des comportements plus autonomes, d'autres se retrouvent dans une situation objective de dépendance matérielle et émotive qui les freine. Les femmes violentées ou les jeunes mères chefs de famille se sentent souvent tellement écrasées qu'elles ne voient aucune porte de sortie. Elles peuvent demeurer dépendantes non seulement dans leur vie quotidienne, mais aussi dans le groupe.

Cette réalité pose une difficulté aux animatrices qui ne veulent pas que le groupe soit dépendant d'elles. D'une part, il faut encourager les membres à devenir graduellement plus autonomes à l'intérieur et à l'extérieur du groupe. Autrement, les animatrices, socialisées elles aussi à toujours être au service des autres, peuvent se sentir coupables de ne pas pouvoir répondre à tous les besoins de chaque femme. D'autre part, il faut accepter que la resocialisation qui produit des comportements autonomes soit difficile et ne survienne pas du jour au lendemain. Les animatrices seront peut-être obligées d'adopter temporairement un rôle plus central et d'attendre que le groupe et ses membres évoluent, avant de se retirer dans un rôle de facilitateures / personnes-ressources. Dans certaines situations où la présence d'une animatrice professionnelle n'est pas prévue, il faut accepter que le groupe ait besoin d'une période de transition pour acquérir assez de confiance et de compétence pour travailler de façon autonome.

L'animatrice professionnelle peut vivre des conflits importants. Sa formation lui suggère d'aider une femme en difficulté, alors que l'idéologie féministe veut qu'elle évite de trop intervenir de peur de nuire à l'autonomie de la femme. Dans un groupe de soutien/ conscientisation auquel l'auteure a participé, une femme a partagé avec les autres son vécu quant au fait d'avoir quitté son mari et ses enfants. Le groupe lui a offert du soutien, reconnaissant qu'elle avait eu beaucoup de courage dans un contexte où la société rejette les femmes qui quittent leur famille. Ayant remarqué qu'une autre femme, qui participait d'habitude, était silencieuse et semblait malheureuse, les animatrices se demandaient si elles devaient intervenir, mais hésitaient. 
Après la réunion, la femme en question a expliqué à un autre membre son malaise, du fait que sa mère l'avait abandonnée quand elle était très jeune. L'autre membre l'a persuadée d'en parler aux animatrices qui l'ont aidée à en discuter en groupe. Cette situation s'est réglée, peutêtre parce que le contrat de départ insistait sur l'importance d'exprimer les insatisfactions plutôt que de quitter le groupe. Mais si cette femme avait quitté le groupe, les animatrices auraient pu vivre beaucoup de culpabilité.

Une façon de faire face au dilemme du rôle à adopter est de choisir la coanimation. Cette option réduit le risque que le groupe dépende d'une personne ou que celle-ci se sente la seule responsable du groupe (WCREC, 1985). Dans l'exemple précité, une animatrice avait une formation professionnelle alors que l'autre avait beaucoup d'expérience dans les centres et les groupes de femmes. Cette dernière freinait les tendances protectrices et interventionnistes de la professionnelle qui, pour sa part, aidait sa collègue à nuancer l'idéologie féministe, à être plus systématique et à accepter le rythme lent du changement. De plus, la coanimation a allégé le fardeau initial d'organiser et d'animer les rencontres. Toutefois, la coanimation n'est pas sans difficultés. Les animatrices doivent s'entendre sur les objectifs, les procédures et surtout le style d'animation. II n'est pas nécessaire d'avoir un style identique ni d'être toujours d'accord; l'existence de différences montre aux membres que personne n'a toutes les réponses et qu'on peut tolérer les dissidences. Cependant, il faut éviter des objectifs et des styles contradictoires. Dans un même groupe, il sera difficile de concilier, par exemple, le rôle central de thérapeute avec le rôle périphérique de personne-ressource, à cause des perceptions sous-jacentes différentes de l'autonomie des membres. Si les coanimatrices sont d'accord sur les objectifs et les principes de base (leur rôle, les normes, etc.), le fait de communiquer ouvertement et de s'allouer du temps de discussion entre les réunions peut réduire les problèmes (Shulman, 1979).

Par ailleurs, le facteur culturel complique la question du rythme de changement vers l'autonomie. D'abord, les animatrices blanches, de classe moyenne, ne peuvent pas toujours présumer que les femmes issues d'autres milieux partagent l'objectif d'autonomie. Deux travailleuses sociales qui ont mis sur pied un groupe de femmes immigrantes en Australie ont constaté que leur définition de l'autonomie était très différente de celle des membres. Avant de recruter certaines femmes arabes, les intervenantes ont dû aller rencontrer les maris pour leur expliquer les objectifs et le programme du groupe afin de les convaincre de laisser leur épouse y participer. Pour elles, pouvoir sortir seules de la maison pour une activité entre femmes, c'était déjà un pas énorme vers l'autonomie. Les animatrices ont alors atténué beaucoup leurs objectifs 
de conscientisation pour axer le groupe sur la diminution de l'isolement et le partage du vécu, afin de tenir compte de l'impact des obstacles culturels pour ces femmes.

La question culturelle influence également la priorité à accorder au soutien ou au défi au changement. Idéalement, les groupes devraient équilibrer les deux buts, car trop de soutien peut produire de l'apathie, alors que trop de pression pour changer peut immobiliser les membres en provoquant de l'anxiété (Northen, 1969). Il faut tenir compte de la disponibilité personnelle au changement et aider chaque membre à choisir des objectifs individuels réalisables, compte tenu de sa situation. Dans les groupes de changement personnel, un contrat individuel devrait accompagner le contrat de groupe. Une femme, d'une origine culturelle prônant des valeurs traditionnelles par rapport au rôle de la femme, est devenue membre d'un groupe d'affirmation de soi. Les animatrices savaient que les femmes de certaines cultures arabes et asiatiques peuvent difficilement devenir plus autonomes à cause des conflits de valeurs. Elles lui ont alors suggéré de commencer à s'affirmer petit à petit en demandant à son mari de lui apporter du thé. La femme a plutôt essayé de provoquer un changement majeur chez son mari et après un échec inévitable, elle a quitté le groupe. Pour cette femme, un groupe de soutien/entraide (comme celui regroupant les femmes immigrantes) aurait probablement été plus bénéfique. Même si on critique les groupes de femmes à cause de l'accent mis sur le soutien, il y a bien des situations où il vaut mieux viser de petits changements plutôt que de risquer des modifications majeures. Les techniques telles que l'analyse sociale, l'encouragement de comportements androgynes et la rétroaction comportementale sont trop menaçantes pour des femmes venant de certains milieux, et il est préférable de se restreindre à des techniques axées sur le soutien comme l'auto-révélation et l'évaluation positive (Russell, 1984).

Par contre, il y a des groupes où les femmes sont prêtes à faire des changements importants, mais elles hésitent à se confronter car elles craignent de provoquer un conflit. D'une part, les femmes ont été socialisées à ne pas exprimer ouvertement leurs sentiments d'agressivité, et d'autre part, l'idéologie féministe rejette le conflit et la compétition entre femmes (Hagen, 1983). Si les groupes n'apprennent pas à faire face aux conflits inévitables, les insatisfactions s'accumulent. Les conflits sont souvent exprimés de façon indirecte (apathie, absences, dépression) dans les groupes de femmes. De plus, l'émergence de conflits survient plus tard que dans les groupes mixtes, parce que les femmes travaillent d'abord le thème d'intimité (relations interpersonnelles) avant de toucher au rapport de force entre membres (Hagen, 1983). Lorsqu'on ne reconnaît pas l'existence du conflit, cette étape difficile peut se prolonger et nuire 
à la productivité du groupe. Au niveau individuel, si les membres ont peur d'exprimer leurs sentiments négatifs ou de se confronter, ils ne mobiliseront pas suffisamment l'influence du groupe pour favoriser des changements. Ils n'oseront pas, par exemple, offrir de la rétroaction comportementale à un membre afin de l'aider à changer son comportement.

Les groupes de femmes peuvent apprendre à faire face au conflit en reconnaissant que des différences et des difficultés sont inévitables et que le conflit peut être productif si on le confronte de façon ouverte. En plus d'encourager des normes d'expression honnête des sentiments, les groupes peuvent se servir d'exercices qui visent à clarifier les valeurs et apprendre à donner une rétroaction spécifique (Hagen, 1983).

Les groupes peuvent réduire les problèmes précités en accordant une attention particulière à l'étape de la planification. L'idéologie féministe suggère que les groupes soient ouverts à toute femme désireuse d'y participer et qui se définit comme ayant besoin du groupe. Mais comme nous l'avons vu, les femmes ne savent pas toujours quelle est la meilleure ressource en rapport avec leurs besoins. Alors que les groupes axés sur la conscientisation (CR) et le changement social fonctionnent mieux lorsqu'ils sont formés de femmes d'origines diversifiées, ceux rassemblant des femmes au vécu similaire sont plus aidants au niveau du changement personnel (Home, 1980). II vaut mieux alors clarifier d'avance les critères de sélection, en incluant la question d'accepter ou non les femmes en crise. Certains groupes, par définition, conviennent à ces femmes, mais d'autres ne peuvent pas toujours répondre aux besoins importants de celles-ci. Un groupe solide peut aider une femme qui vit une crise temporaire au cours de l'expérience en lui offrant soutien, acceptation inconditionnelle, conseils ou aide concrète. Par contre, certaines femmes ayant besoin d'une thérapie choisissent plutôt de faire partie d'un groupe de soutien ou de conscientisation (CR), à cause de l'étiquette souvent reliée à la thérapie. Une entrevue avant le début des rencontres peut aider ces femmes à se réorienter sans se sentir rejetées, évitant ainsi le risque d'être plus tard expulsées du groupe.

\section{Conclusion}

Dans cet article, nous avons discuté de l'utilité des groupes de femmes pour promouvoir le changement personnel et le développement. Ces groupes mettent l'accent sur le partage du vécu, la résolution de problèmes vécus par les membres ou la poursuite de croissance personnelle. Les groupes de soutien/entraide insistent sur l'aide mutuelle 
entre les membres comme outil principal de changement, alors que les groupes de thérapie/croissance sont animés par des intervenantes professionnelles qui emploient, entre autres, les techniques d'intervention féministe. Certains autres combinent les techniques et structures de ces groupes avec celles des groupes de conscientisation (CR).

Même si ces groupes offrent aux femmes une alternative intéressante aux services traditionnels, ils peuvent rencontrer des problèmes reliés aux contradictions entre une idéologie féministe parfois idéaliste et la réalité quotidienne des femmes qui en font partie. Ces groupes doivent contourner des difficultés reliées à l'enjeu de l'autonomie versus la dépendance et les conséquences qui en découlent par rapport au rôle du leader, au rythme du changement et à l'équilibre à établir entre le soutien et le défi. De plus, les problèmes posés par les différences culturelles entre femmes continuent de représenter un défi pour les féministes qui veulent regrouper les femmes.

Le nombre et la diversité de groupes de femmes ne cessent d'augmenter. La pratique auprès de ceux-ci devient plus systématique en même temps qu'on identifie plus clairement les techniques et les structures susceptibles de favoriser le changement personnel et le développement. Toutefois, très peu de ces groupes font l'objet de documentation et encore moins sont évalués pour déterminer leur impact. Leur potentiel restera inexploité tant que le partage de connaissances et d'expériences ne dépassera pas le niveau d'échange informel et local. Ces expériences, innovatrices et prometteuses, doivent étre accessibles à d'autres femmes qui ont besoin d'en connaître davantage.

\section{Note}

1 Nous utilisons le terme " groupe de conscientisation " pour décrire des groupes de femmes qui ont comme objectif explicite de promouvoir une prise de conscience de l'oppression vécue par les femmes. Étant donné que le terme original est " consciousness-raising (CR) ", nous avons ajouté " $C R$ " pour éviter toute confusion avec des groupes qui recherchent la prise de conscience par rapport à d'autres types d'oppression.

\section{Références bibliographiques}

ADOLPH, M., "The all women's consciousness-raising group as a component of treatment for mental illness", Social Work with Groups, vol. 6, nos 3-4, 1983 : 117-131. 
ARIES, E., "Interaction patterns and themes of male, female and mixed groups", Small Group Behavior, vol. 7, no 1, 1976 : 7-18.

BERNIER, S., "Analyse d'une nouvelle pratique sociale et féministe auprès des femmes qui font un retour au marché du travail ", Essai de maîtrise non publié, Québec, Université Laval, 1984.

BORKMAN, T., "Experiential knowledge : A new concept for analysis of self-help groups ", Social Service Review, n० 50, 1976 : 445-456.

BUNCH, C., "Self-definition and political survival ", Quest : A feminist Quarterly, no 1, $1975: 2-6$.

BUtLer, P., "Assertive training: Teaching women not to discriminate against themselves ", Psychotherapy: Theory, research and practice, vol. 13, n० 1, 1976 : 56-60.

CARDin, M. et A. HOME, "La pratique du service social avec les groupes de femmes ", Service social, vol. 32, nos 1-2, 1983:170-185.

Corbeil, C., A. Pâquet-Deehy, C. Lazure et G. Legault, L'Intervention féministe, Montréal, Éditions coopératives Albert Saint-Martin, 1983.

Darveau-Fournier, L. et A. Home, "Les groupes de service social à Québec: recherche des rapports entre théorie et pratique ", Service social, vol. 32, nos 1-2, $1983: 129-155$.

DeMERS, M., " Rapport de stage en intervention féministe : femmes et dépression ", texte inédit, Montréal, Université de Montréal, 1984.

DenMARK, F. "Styles of leadership ", Psychology of Women Quarterly, vol. 2, n 2, $1977:$ :99-113.

EICHENBAUM, L., "The effects of same-sex versus mixed-sex assertion training groups on assertiveness, sex-role attitudes and locus of control beliefs in women ", Dissertation Abstracts International, vol. 39, no 05-B, 1978 : 2493.

Farquharson, A., Peers as Helpers: Personal Change in Members of Self-Help Groups in Metropolitan Toronto, thèse de doctorat non publiée en éducation, Toronto, Université de Toronto (Ontario Institute of Studies in Education), 1975.

Freire, P., Pedagogy of the Oppressed, traduit par Myra Bergman Ramos, New York, Herder \& Herder, 1970.

GINGRAS, P., "Intervention auprès d'un groupe de femmes de classe populaire", Service social, vol. 32 , nos 1-2, $1983: 89-100$.

GingRAS, P., La prise en charge des femmes par les femmes: le travail de groupe, un moyen, Conférence prononcée à Rouyn-Noranda le 6 avril 1986.

GLASER, K., "Women's self-help groups as an alternative to therapy », Psychotherapy : Theory, practice and research, vol. 13, no 1, 1976:77-81.

Gottlieb, N., D. BURden, R. MCCORMick et G. NiCARTHY, "The distinctive attributes of feminist groups", Social Work with Groups, vol. 6, nos 3-4, $1983: 81-93$.

HAGEN, B., "Managing conflict in all-women groups", Social Work with Groups, vol. 6 , nos 3-4, 1983: 95-104.

HartmanN, S., "A self-help group for women in abusive relationships", Social Work with Groups, vol. 6, nos 3-4, 1983 : 133-146. 
HAUSSMANN, M. et J. HALSETH, "Re-examining women's roles: A feminist group approach to decreasing depression in women ", Social Work with Groups, vol. 6 , nos 3-4, $1983: 105-115$.

HÉBERT, L., Utilisation complémentaire de l'intervention féministe et du modèle de réciprocité dans un groupe de femmes aînées, Essai inédit, Québec, Université Laval, 1985.

HOME, A., "Étude exploratoire de quatre types de changements survenus chez les membres de groupes féminins de conscientisation", Service social, vol. 29, nos 1-2, 1980 : 152-181.

HOME, A., "Intervention with groups", dans: S. YeLAJA (éd.), An Introduction to Social Work Practice in Canada, Toronto, Prentice-Hall, 1985.

HOME, A., "Les femmes et les groupes de changement social", Service social, vol. 32, nos 1-2, $1983:$ 50-78.

JAKUBOWSKI-SPECTOR, P., An Introduction to Assertiveness Training Procedures for Women, Washington, American Personnel and Guidance Association, 1973.

KiLleEN, M. et C. JACOBS, "Brief group therapy for women students ", Social Work, vol. $21, n^{\circ} 6,1976: 521-522$.

KUSH-GOLDBERG, C., "Health self-help group as an alternative source of health care for women ", International Journal of Nursing Studies, vol. 16, no 3, 1979 : 283-294.

LABROSSE, J., L'intervention féministe : théorie et application, Rapport de stage inédit, Montréal, Université de Montréal. 1987.

LANG, N., "A broad-range model of practice in the social work group", Social Service Review, vol. 40, 1972 : 76-89.

Larouche, G. et L. Vaillant, Bilan du groupe Entr'elles VI, Montréal, Centre de services sociaux du Montréal métropolitain, 1986.

LARSEN, C. et L. CAMMAerT, "Feminism at the grassroots", dans : L. Rosewater et $L$. WALKER, Handbook of Feminist Therapy: Women's Issues in Psychotherapy, New York, Springer, 1985.

LEVENS, H. "Organizational affiliation and powerlessness: A case study of the welfare poor", Social Problems, vol. 16, 1968 : 18-32.

LEVIN, S., A. GROVES et J. LURIE, "Sharing the move - support groups for relocated women ", Social Work, vol. 25, no 4, $1980: 323-325$.

Levy, L., "Processes and activities in groups", M. Lieberman et L. Borman, SelfHelp Groups for Coping with Crisis, San Francisco, Jossey-Bass, 1979 : 234-271.

MARACEK, J., "Social change, positive mental health and psychological androgyny ", Psychology of Women Quarterly, vol. 3, n 3, 1979: 241-247.

MAUSERT, R., "The impact of women's groups upon psychological androgyny", Masters Abstracts, vol. 18, no 3, 1979, p. 237.

MEAdor, B., E. SOlomon et M. Bowen, "Encounter groups for women only", dans: L. Solomon et B. Berzon (éds.), New Perspectives on Encounter Groups, San Francisco, Jossey-Bass, 1972: 335-348.

NORTHeN, H., Social Work with Groups, New York, Columbia University, 1969.

Redfern, M., S. Klass, et K. Coleman, "A housewive's group in a university setting ", Social Work, vol. 21, n 6, $1976: 527-528$. 
ReisSman, F., "The "helper therapy" principle ", Social Work, vol. 10, $1965: 27-32$.

ROSENBERG, P., "Support groups: A special therapeutic entity", Small Group Behavior, vol. 15, no 2, 1984 : 173-186.

Rotrman, J., Planning and Organizing for Social Change, New York et Londres, Columbia, University Press, 1974.

Rounsaville, B., N. LifTon et M. BieBer, "The natural history of a psychotherapy group for battered women ", Psychiatry, vol. 42, 1979:63-78.

RusselL, M., Skills in Counseling Women, Springfield, Charles C. Thomas, 1984.

SHULmAN, L., The Skills of Helping Individuals and Groups, Itasca, Peacock, 1979.

SPREI-OTT, J., "The use of assertiveness training and counsciousness-raising groups in the treatment of depression in women ", Dissertation Abstracts International, vol. 40, 10, 1980, p. 5024.

StURDivant, S., Therapy with Women, New York, Springer, 1980.

TODER, N., "The effect of the sexual composition of a group on discrimination against women and sex-role attitudes ", Psychology of Women Quarterly, vol. 5, no 2, $1980: 292-310$.

VANDERVOET, S. et al., Opportunity for Advancement, Gouvernement du Canada, Santé et bien-être social, Project for sole support mothers on government assistance, Toronto, 1978.

WALKER, L., "Are women's groups different ? ", Psychotherapy: Theory, research and practice, vol. 18, $\mathrm{n}^{\circ} 2,1981: 240-245$.

WCREC, A Handbook for Women Starting Groups, Toronto, Women's Press, 1985.

WHEELAN, S., "Sexism in personal growth groups : A partial remedy ", Group and Organization Studies, vol. 5, 1980 : 105-112.

WILMUTH, P., "Effects of sex composition of the group on assertion training outcome", Dissertation Abstracts International, vol. 41, no 5, 1979 : 18981899.

WODAK, R., "Women relate, men report : Sex differences in language behavior in a therapeutic group ", Journal of Pragmatics, vol. 5, no 2-3, 1981 : 261-285.

Wolman, C., "Therapy groups for women", American Journal of Psychiatry, vol. 133, no 3, $1976: 274-278$.

Women and Mental Health Project, "Women-to-women services ", Social Policy, vol. 7, n० 2, $1976: 21-27$.

WrCKoff, H., "Radical psychiatry in women's groups", dans J. AGel (éd.), The Radical Therapist, New York, Ballantine Books, 1971 : 181-187.

Wrckoff, H., Solving Women's Problems, New York, Grove Press, 1977. 